\title{
Enfermedades y padeceres por los que se recurre a terapeutas tradicionales de la Ciudad de México
}

\author{
Shoshana Berenzon-Gorn, Dra, (1) Emily Ito-Sugiyama, Dra, ${ }^{(2)}$ Luis Alberto Vargas-Guadarrama, Dr. ${ }^{(3)}$
}

\begin{abstract}
Berenzon-Gorn S, Ito-Sugiyama E, Vargas-Guadarrama LA. Enfermedades y padeceres por los que se recurre a terapeutas tradicionales de la Ciudad de México. Salud Publica Mex 2006;48:45-56.
\end{abstract}

\section{Resumen}

Objetivo. Presentar los principales motivos de consulta con terapeutas tradicionales de la Ciudad de México. Material y métodos. Se tomó como base los relatos de 16 médicos tradicionales de la Ciudad de México y 22 usuarios de este tipo de medicina. Los métodos utilizados para la recolección y análisis de los datos son de corte cualitativo. El trabajo de campo se realizó en dos etapas. Durante la primera fase se hicieron observaciones con enfoque etnográfico; y en la segunda, se realizaron entrevistas focalizadas. Resultados. De acuerdo con la percepción de los entrevistados, entre los principales motivos de consulta se encuentran los problemas amorosos, conflictos familiares, apuros económicos, estrés, tensión, nervios y angustias espirituales. Estos motivos de consulta no se pueden englobar como enfermedades en un sentido estricto, todos son problemas de salud que se manifiestan como padecimientos y solamente algunos se ubican en la categoría biomédica de enfermedades. Las personas que acuden con los terapeutas tradicionales no solamente buscan curarse de sus males, también desean obtener consejos acerca de sus problemas personales. Discusión. La información analizada en este trabajo permite señalar que existen diversas razones para explicar no solamente la supervivencia de la medicina tradicional, sino además su éxito en la vida actual, donde la ciencia y la tecnología han obtenido resultados sobresalientes, pero a cambio, la biomedicina ha olvidado la complejidad de la vida humana cotidiana y el valor de la atención personal, la compasión, la comprensión, la empatía y el uso del mundo simbólico para la solución de los conflictos y problemas de salud propios de la humanidad.

Palabras clave: enfermedades y padeceres; terapeutas tradicionales; medicina tradicional; Ciudad de México
Berenzon-Gorn S, Ito-Sugiyama E, Vargas-Guadarrama LA. Diseases and illnesses for which help is sought from traditional healers in Mexico City.

Salud Publica Mex 2006;48:45-56.

\section{Abstract}

Objective. The aim of this paper is to describe the main reasons why people consult traditional healers in Mexico City. Material and methods. The narrative of 16 traditional healers and 22 users of this type of medicine is the main source of information. This study used a qualitative approach. The field work was carried out in two stages. The first phase involved a series of observations with an ethnographic approach. During the second phase, focused interviews were held with selected informants. Results. According to participants, the main reasons for consulting traditional healers were problems with romance and love, family conflicts, economic hardships, stress, tension, nervousness and spiritual distress. These motives cannot be considered diseases in a strict sense; they were all health problems manifested as illnesses that caused suffering and only some of them could be classified as diseases within the biomedical model. People go to traditional healers not only to cure their diseases but also to obtain advice about their personal problems. As a result, traditional medicine becomes an additional option for treating human suffering, not only physical, but moral and psychological as well. Discussion. The information analyzed in this paper indicates that diverse reasons exist which explain not only the survival of traditional medicine, but also its importance in present life, where science and technology have obtained outstanding results, but biomedical medicine has forgotten the complexity of daily human life and the importance of personal attention, compassion, understanding, empathy and the use of the symbolic world to solve the conflicts and health problems characteristic to humanity.

Key words: diseases and illnesses; traditional healers; traditional medicina; Mexico

(I) Instituto Nacional de Psiquiatría Ramón de la Fuente, México.

(2) Facultad de Psicología Universidad Nacional Autónoma de México (UNAM).

(3) Instituto de Investigaciones Antropológicas y Facultad de Medicina UNAM.

Fecha de recibido: 18 de febrero de 2005 - Fecha de aprobado: 3 de noviembre de 2005 Solicitud de sobretiros: Dra. Shoshana Berenzon Gorn. Instituto Mexicano de Psiquiatría "Ramón de la Fuente Muñiz". Camino a Xochimilco I0I, Col. San Lorenzo Huipulco, Deleg. Tlalpan. I4370 México, D.F., México. Correo electrónico: berenz@imp.edu.mx 
E propósito del presente trabajo es conocer y analizar el tipo de enfermedades y padecimientos más frecuentes por los que acuden las personas con terapeutas tradicionales de la Ciudad de México, a partir de la información relatada por 16 médicos tradicionales y 22 usuarios de este tipo de medicina.

\section{Panorama mundial sobre el uso de la medicina tradicional}

En diversos estudios se ha puesto en evidencia que la medicina tradicional $^{*}{ }^{1-2}$ se utiliza ampliamente en las distintas regiones del mundo para el tratamiento de un importante número de enfermedades y padecimientos. En África, aproximadamente $80 \%$ de la población recurre a la medicina tradicional para resolver sus problemas de salud. En Asia y Latinoamérica, las poblaciones siguen utilizando la medicina tradicional como resultado de circunstancias históricas y creencias culturales. En China, 40\% de la atención sanitaria está a cargo de la medicina tradicional. ${ }^{2}$ Pese a que en América Latina sólo existen datos disponibles para Chile, Colombia, Bolivia y Cuba, ${ }^{1}$ Viesca $^{3}$ estima que en los países latinoamericanos más de $50 \%$ de la población sigue atendiéndose y percibiendo sus problemas de salud de acuerdo con los patrones de su medicina tradicional.

Diversos estudios han documentado que la medicina tradicional y las terapias alternativas o complementarias $^{\ddagger}$ son utilizadas principalmente por personas

\footnotetext{
* Según la OPS, la medicina tradicional comprende el conjunto de ideas, conceptos, creencias, mitos y procedimientos, sean explicables o no, relativos a las enfermedades físicas, mentales o desequilibrios sociales en un pueblo determinado. Este conjunto de conocimientos explican la etiología, la nosología y los procedimientos de diagnóstico, pronóstico, curación y prevención de las enfermedades; se transmiten por tradición y verbalmente de generación en generación, dentro de los pueblos. Lo anterior significa que esta medicina es circunscrita, local, colectiva y anónima (referencia 1).

Desde una visión más práctica, la OMS define a la medicina tradicional como prácticas, enfoques, conocimientos y creencias sanitarias diversas que incorporan medicinas basadas en plantas, animales y/o minerales, terapias espirituales, técnicas manuales y ejercicios aplicados de forma individual o en combinación para mantener el bienestar, además de tratar, diagnosticar y prevenir las enfermedades (referencia 2).

₹ Los términos alternativas o complementarias se refieren a un amplio grupo de prácticas sanitarias que no forman parte de la tradición de un propio país, o no están integradas al sistema sanitario dominante, pero que son practicadas y utilizadas por un grupo importante de la población. Un ejemplo de lo anterior sería la incorporación de la acupuntura, el yoga o el feng shui entre la población mexicana. Si bien son saberes y prácticas tradicionales en China o en la India, en México ingresan de manera diferente al ser desligados del campo social y cultural donde fueron producidos (referencias 2 y 4 )
}

que sufren enfermedades crónicas como cáncer, artritis, problemas gastrointestinales, esclerosis múltiple o enfermedades dermatológicas. Otras razones para su empleo están asociadas con la idea de prevenir enfermedades y mantener la salud, ${ }^{5-8}$

En la literatura internacional también se sugiere un elevado uso de la medicina tradicional y las terapias alternativas por personas que presentan padecimientos emocionales, sobre todo los que sufren problemas depresivos y de ansiedad. En dos encuestas telefónicas realizadas en Estados Unidos de América se constató que entre 40 y $55 \%$ de las personas que presentan ataques de ansiedad o depresión severa utilizan terapias alternativas en combinación con la medicina ortodoxa. ${ }^{5,9}$

De acuerdo con la Organización Panamericana de la Salud, en los países latinoamericanos se recurre a la medicina tradicional por una gran diversidad de problemas, entre los que resaltan: infecciones estomacales, accidentes, envidia, susto, caída de la mollera, nervios, problemas músculo-esqueléticos, factores económicos y haber experimentado fracasos durante la atención por la medicina alopática. ${ }^{2}$

Investigaciones tanto de corte cuantitativo como cualitativo plantean que una de las principales razones para la consulta a las medicinas tradicionales y/o a las alternativas o complementarias es el obtener beneficios terapéuticos. Entre los factores que influyen sobre esta decisión se encuentran: el deseo de evitar la toxicidad, los métodos invasivos o los efectos secundarios; o bien, alcanzar niveles de calidad de vida que no son posibles con las terapias convencionales. Otras de las razones son: el encontrar las teorías médicas institucionales simplistas, incompletas o inadecuadas; la facilidad de acceso y costos moderados de los servicios; la curiosidad nacida de anécdotas o testimonios por parte de amigos y familiares que ya han asistido; el pensar que van a recibir un trato más cordial y cercano que el otorgado por el médico alópata; y el buscar involucrase más en la toma de decisiones, tanto de la enfermedad como de los tratamientos a seguir., 60 -13

\section{La medicina tradicional en el contexto urbano mexicano}

Lo que hoy llamamos medicina tradicional en México es en realidad una abstracción que reúne varios sistemas de salud de los cuales la mayoría tienen algunos elementos comunes. El primero es tener como antecedentes históricos a las medicinas de los indígenas mesoamericanos, así como la española del siglo XVI, de las que perviven algunos conceptos y prácticas. El segundo es haberse enriquecido en un grado variable 
con aportaciones de otros sistemas, como la de los esclavos llegados de África, la medicina científica contemporánea y otras de reciente introducción, por ejemplo, algunos elementos de la medicina tradicional china. El tercero es ser sumamente dinámicas para adoptar recursos que les ofrece el ambiente, por lo que alguno de sus practicantes podría combinar el empleo de antibióticos o una pomada corticoides con conceptos del alma de origen mesoamericano, una ceremonia durante la que cae en trance, al estilo africano, la aplicación de agujas de acupuntura o recomendaciones derivadas del feng shui. Una característica más de la medicina tradicional es su subordinación formal ante la medicina oficial, académica o científica, al grado de no estar de manera formal plenamente reconocidas desde el punto de vista jurídico, a pesar de su popularidad.

Debido a que esta investigación se desarrolló en un medio urbano, es importante mencionar que existe una gran diversidad de factores que diferencian a la medicina tradicional urbana de la rural. Redfield ${ }^{14}$ y Campos $^{12}$ señalan que el curandero urbano difiere del rural en que no ocupa un lugar privilegiado dentro de la comunidad, debido a que sus funciones se restringen a la práctica curativa. Sostiene rivalidades frecuentes con otros curanderos urbanos y muestra una clara comercialización de sus actividades terapéuticas. Aunado a lo anterior, el curandero urbano, a diferencia del rural, se diversifica y se especializa para hacer frente a la heterogeneidad de padecimientos que atiende y, por ello, tiene una apertura ideológica ante nuevas prácticas y conocimientos médicos. ${ }^{15}$

Trotter y Chavira ${ }^{16}$ consideran que dados los recursos sanitarios y de atención médica que existen en la actualidad en los medios urbanos, ha cambiado la función del curandero: se recurre a él principalmente por problemas de salud mental. En este mismo orden de ideas, Campos ${ }^{13}$ sugiere que los destructores efectos emocionales o psicológicos de la vida moderna han dado posiblemente al curanderismo urbano un significado y una función más especializada. Por ejemplo, funcionan como válvula de escape y alivio a las tensiones que se generan cotidianamente en los sectores urbanos.

También existen diferencias en cuanto a los motivos de consulta. En las zonas rurales de la República mexicana se recurre a los terapeutas tradicionales principalmente por fiebre, diarrea, problemas de la piel, tos intensa, dolor de garganta, nervios, mal de ojo, susto, empacho y caída de mollera; las causas de demanda en las urbanas son: infecciones gastrointestinales, accidentes, problemas de envidia, mal echado o puesto, susto, insomnio, nervios, depresión y enfermedades "que no curan los médicos". ${ }^{1}$

\section{Enfermedad y padecer}

La antropología médica norteamericana distingue por lo menos dos dimensiones de la misma enfermedad: enfermedad (disease) y padecimiento (illness). La enfermedad se refiere a la anormalidad en la estructura y / o función del órgano o sistema de órganos, pertenece al campo del modelo biomédico. Es la forma en que los practicantes de la medicina oficial interpretan un problema de salud. El padecimiento es la experiencia subjetiva de los síntomas y del sufrimiento, es decir, cómo la persona enferma los presenta, qué perciben los miembros de su familia o de la comunidad, cómo viven y reaccionan frente a los síntomas y a las incapacidades que estos generan. ${ }^{17}$ Los padecimientos se refieren al conjunto de conceptos, experiencias y sentimientos comunes de un determinado grupo social, constituidos de manera múltiple, y que relacionan el contexto social e histórico con la experiencia biopsíquica. ${ }^{17,18}$

\section{Material y métodos}

A continuación se desglosan las técnicas utilizadas tanto para la búsqueda y el registro de la información como para el análisis y la interpretación de los datos. Todas las técnicas están sustentadas en los supuestos teóricos interpretativos, donde se destaca la capacidad de los actores sociales para dar significado a diferentes situaciones sociales y de actuar y no sólo de reaccionar en consecuencia.

El trabajo de campo de esta investigación se realizó en dos etapas interrelacionadas: durante la primera fase se hicieron una serie de observaciones con enfoque etnográfico y en la segunda se realizaron entrevistas focalizadas. ${ }^{19}$ El trabajo de campo se inicio en noviembre de 1999 y se concluyó en diciembre de 2001.

\section{Población}

El estudio se llevó a cabo con 16 terapeutas tradicionales que brindan sus servicios en distintos puntos de la Ciudad de México y con 22 personas que recurren a los mismos. Para la selección de los informantes se utilizó la estrategia de muestreo teórico, propuesto por Glasser y Strauss, ${ }^{20}$ donde lo más importante no es el número de personas entrevistadas sino la información que proporcionen para interpretar el tema de interés. Mediante este tipo de muestreo se desea obtener información que complemente lo que se está investigando, que resuelva contradicciones o que añada aquellas partes del proceso que todavía no se han observado. El proceso termina cuando las entrevistas con perso- 
nas adicionales no producen ninguna información nueva o relevante para la comprensión del fenómeno; este proceso se conoce como saturación.

\section{Aproximación a los informantes}

El contacto con los entrevistados se hizo a través de la técnica de "bola de nieve", que es un caso particular de análisis de cadenas que intenta construir una muestra de individuos que comparten características comunes en un universo más amplio. ${ }^{21}$ En este caso, el primer curandero entrevistado fungió como agente de búsqueda de casos e introdujo a los investigadores con otros curanderos que, a su vez, acercaron a sus pacientes.

Una vez establecido el contacto tanto con los terapeutas tradicionales como con los pacientes, los investigadores hicieron una presentación, a través de la cual se estableció una relación lo más honesta posible y de confianza mutua. Para lo anterior, al entrevistado se le explicaron los motivos de la entrevista, el carácter confidencial de los datos y se le pidió autorización para grabar las entrevistas y para la divulgación de la información recolectada. ${ }^{*}, 22$ El consentimiento informado de las personas que participaron en el proyecto se obtuvo de manera verbal.

El comité de ética en investigación del Instituto Nacional de Psiquiatría Ramón de la Fuente aprobó el protocolo de investigación "Estudio sobre recursos alternativos o informales a los que acude la población con problemas de salud mental en una zona sur de la Ciudad de México", proyecto del cual provienen los resultados que se presentan más adelante.

\section{Realización del estudio}

\section{Primera fase: estudio etnográfico}

En cada uno de los recorridos de campo realizados a lo largo de esta investigación, se hicieron observaciones que se registraron por medio de notas de campo. ${ }^{23}$ Las características de las observaciones se tomaron de la propuesta que hace Galindo sobre las fases del trabajo etnográfico. ${ }^{24}$ Se hicieron observaciones exploratorias y descriptivas. Las observaciones exploratorias permitieron tener un conocimiento general de las zonas en donde estaban localizados los "consultorios" de

\footnotetext{
* Los cuidados éticos fundamentales de la investigación cualitativa son tres: el consentimiento con información previa, la protección de la confidencialidad de la información y el resguardo del uso abusivo del poder en la relación entre el investigador y participantes (referencia 22).
}

los médicos tradicionales. En las observaciones descriptivas, el registro fue mucho más detallado. Se incluyeron las características de los médicos tradicionales, los pacientes y los consultorios. Se registraron las curaciones que se pudieron observar, la preparación de los remedios y las indicaciones dadas a los pacientes.

Segunda fase: entrevistas

Se realizaron entrevistas focalizadas utilizando la técnica de entrevista de investigación social. ${ }^{* 27}$ Para recopilar la información se elaboraron dos guías de entrevista, una para los terapeutas tradicionales y otra para los pacientes. Para cubrir el objetivo de este artículo, sólo se analizaron los principales motivos de consulta con la medicina tradicional desde el punto de vista tanto de los terapeutas como de los pacientes. De la guía de entrevista de los terapeutas tradicionales se incluyeron preguntas relacionadas con las razones por las que creen que acuden las personas a solicitar sus servicios, procedimientos utilizados para clasificar y determinar el tipo de enfermedad y descripción y definición de los padecimientos. Los temas incluidos de los pacientes fueron: motivos de consulta, forma en que definen los padeceres y descripción de los mismos (cuadro I).

\section{Análisis de la información}

Todas las entrevistas se transcribieron en texto para posteriormente confrontar los registros auditivos con los registros en papel y corregir los posibles errores. Se elaboraron categorías para clasificar la información de los temas tratados en las conversaciones. Estas categorías se identificaron con base en los propósitos propuestos en las guías de entrevistas y se agruparon utilizando la técnica de categorización de significados propuesta por Kvale. ${ }^{\ddagger}, 25$ Con el propósito de evitar po-

\footnotetext{
* A través de esta técnica se busca, a partir de un conjunto de saberes privados, la construcción del sentido social de la conducta individual o del grupo de referencia del individuo. De este modo, la entrevista de investigación es una conversación entre dos personas (entrevistador e informante), dirigida y registrada por el entrevistador, con el propósito de favorecer la producción de un discurso conversacional, continuo y con una cierta línea argumental, sobre un tema determinado en el marco de la investigación (referencia 27).

₹ Por medio de esta técnica, cada entrevista se codifica en una serie de categorías mutuamente excluyentes, lo que permite estructurar las extensas y complejas narraciones en unidades de información que facilitan la comprensión de un determinado fenómeno, así como su ocurrencia a lo largo de la entrevista, además de facilitar la descripción de las características más importantes del contenido.
}

salud pública de méxico / vol.48, no.1, enero-febrero de 2006 


\section{Cuadro I \\ Temas clave de las guías de entrevista}

Médicos tradicionales

Temas clave

I) Historia personal relacionada con su participación en esta actividad.

2) Descripción de su vida actual dentro de esta actividad (características del servicio que otorga, tratamientos utilizados).

3) Percepción que tiene del trabajo que realiza.

4) Percepción sobre las personas que acuden a su servicio (características, lugares de dónde acuden, motivos de consulta, etc.)

5) Remuneración de los servicios.

6) Percepción social y cultural de la salud mental.

7) Percepción social y cultural de su actividad.

8) Relación con otros posibles servicios de atención.

9 Datos sociodemográficos.
Pacientes

Temas clave

I) Historia clínica del padecimiento.

2) Trayectoria de búsqueda de atención a su problema de salud (personas o servicios a los que acudió, periodo y orden en que asistió, razones para recurrir o no con las diferentes personas o servicios); diferencias y similitudes entre las personas y servicios visitados.

3) Asistencia con el curandero (primer contacto con el curandero; tratamientos recibidos, percepciones y sensaciones sobre el tratamiento recibido).

4) Percepción social y cultural de la salud mental.

5) Datos sociodemográficos.

sibles discrepancias de criterio durante este proceso, dos personas por separado codificaron el mismo material y posteriormente se compararon las categorizaciones obtenidas por cada una. Las discrepancias en la codificación fueron resueltas con la discusión y con una revisión conjunta de los relatos originales. ${ }^{25}$

El siguiente paso consistió en realizar una categorización más fina del tema. A diferencia del primer proceso de codificación que se hizo de manera deductiva (los temas fueron determinados a partir del objetivo del trabajo), la codificación más fina de cada tema se desarrolló de forma inductiva, ya que las sucesivas lecturas de cada entrevista fueron lo que permitió reconocer qué información había en cada una. Este proceso de codificación permitió construir las categorías que mejor se adaptaban a la información recopilada, lo que a su vez facilitó la interpretación y análisis de los testimonios a partir de los referentes teóricos. ${ }^{26}$

Posteriormente, las categorías generadas se estudiaron en conjunto con todo el texto y se analizó de qué forma cada entrevista estaba determinada por la cultura de los informantes, por la sensibilidad y conocimiento del tema y por la relación espacial y / o temporal en que se llevó a cabo la entrevista. Es decir, se estudió el discurso de los entrevistados en su contexto. ${ }^{27}$

\section{Resultados}

\section{Los entrevistados}

Se entrevistaron 16 médicos tradicionales (ocho hombres y ocho mujeres) que residen en la Ciudad de México y 22 personas (siete hombres y 15 mujeres) que asistieron con un médico tradicional. Con la finalidad de respetar la confidencialidad de la información se utilizó un nombre ficticio para cada uno de los infor- mantes. Las principales características sociodemográficas de los terapeutas tradicionales se muestran en la cuadro II y las características de los usuarios en el cuadro III.

Es importante mencionar que para los propósitos del trabajo, se intentó hacer una clasificación de los terapeutas de acuerdo con su especialidad, determinada, sobre todo, por las características de la terapéutica utilizada. La mayoría informó que manejaba diversas modalidades terapéuticas al mismo tiempo, por lo que se respetó el término con el que ellos se autonombraban (cuadro II).

\section{Principales motivos de consulta}

Para facilitar la descripción de la información recolectada en relación con el tipo de problemas de salud por los que se recurre a la medicina tradicional, se elaboraron las siguientes tipologías o clasificaciones: a) problemática asociada con el cotidiano vivir; b) el estrés, la tensión y los nervios; c) padecimientos musculoesqueléticos; d) enfermedades relacionadas con los diversos sistemas que regulan el cuerpo; e) problemas espirituales; f) enfermedades provocadas y g) padecimientos asociados con la identidad cultural de hombres y de mujeres.

\section{Problemas asociados con el cotidiano vivir}

De acuerdo con la percepción de los médicos tradicionales y de los usuarios, entre los principales motivos de consulta se encuentran los problemas amorosos (conseguir marido, novio, alejar al amante); los familiares (peleas con los padres, los hijos, los yernos y nueras); los laborales (conseguir trabajo, que salga algún negocio, promoción o ascenso en el trabajo actual); los 


\title{
Cuadro II
}

\section{Características sociodemográficas de los médicos tradicionales, México}

\author{
Edad Residencia actual Grado máximo de estudios Ocupaciones actuales manifestadas
}

Carlos

\begin{tabular}{lllll} 
Curandero* & 38 años & Ciudad de México & Licenciatura & Curandero \\
\hline $\begin{array}{l}\text { José } \\
\text { Curandero* }\end{array}$ & 27 años & Ciudad de México & Secundaria & Tiene un negocio de hierbas y veladoras \\
\hline $\begin{array}{l}\text { Pablo } \\
\text { Espiritualista }{ }^{\S} \text { y yerbero }\end{array}$ & 34 años & Ciudad de México & Secundaria & Espiritualista y yerbero \\
$\begin{array}{l}\text { Manuel } \\
\text { Curandero* }\end{array}$ & 33 años & Ciudad de México & Preparatoria & Curandero \\
\hline
\end{tabular}

Fernando

Médico tradicional* 44 años Ciudad de México Secundaria $\quad$ Medicina tradicional e instructor de

danza tradicional azteca

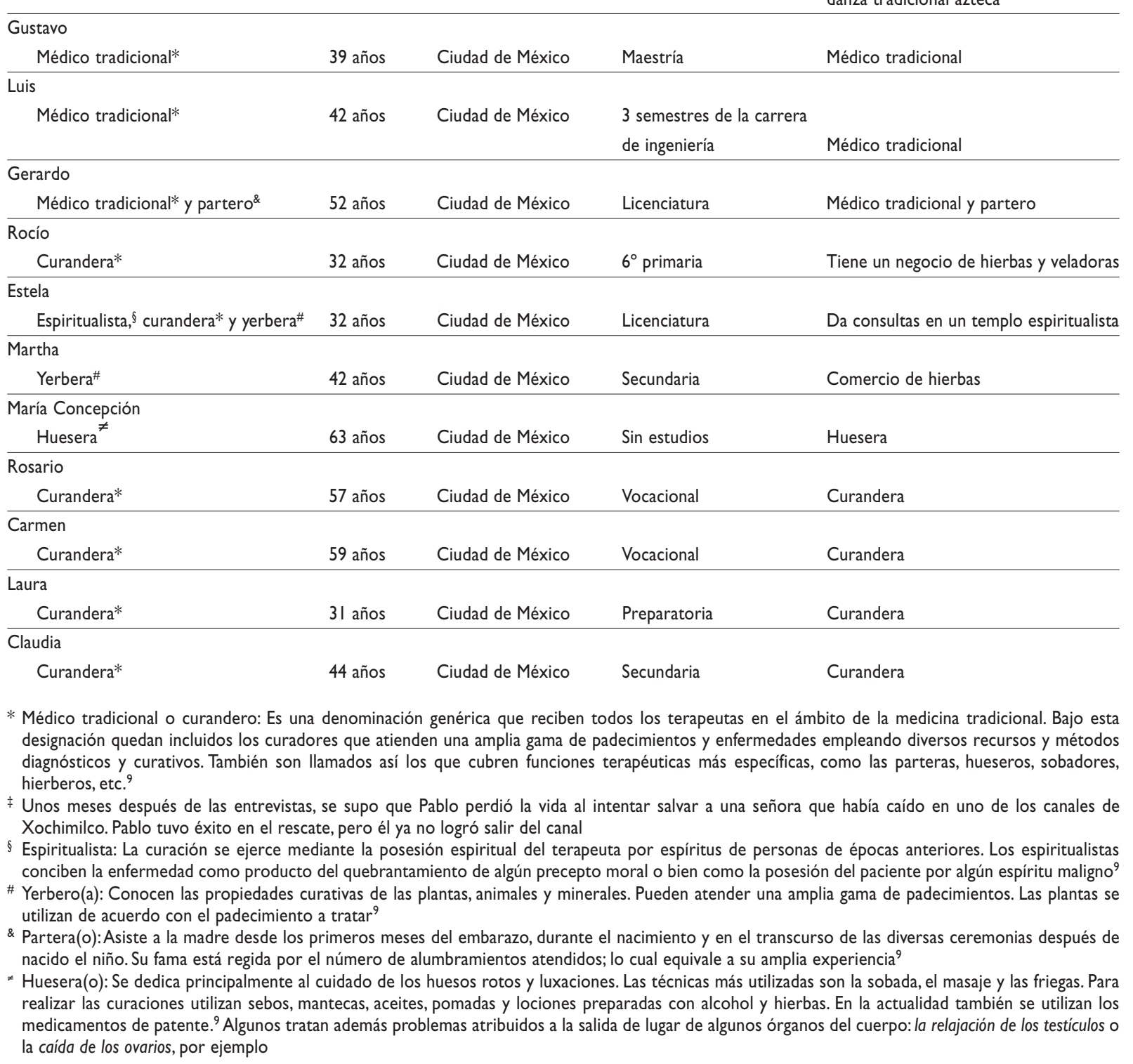




\section{Características sociodemográficas de los pacientes, México}

\begin{tabular}{|c|c|c|c|c|c|}
\hline & Edad & Grado máximo de estudios & Ocupación & Estado civil & Religión \\
\hline Claudia & 40 años & Licenciatura & Cantante & Soltera & Ninguna \\
\hline Rosa & 78 años & Licenciatura & Periodista & Viuda & Católica \\
\hline Mercedes & 58 años & Licenciatura & Médico & Casada & Católica \\
\hline Mónica & 36 años & Licenciatura & Empresa privada & Casada & Católica \\
\hline María & 43 años & Posgrado & Enfermera & Casada & Católica \\
\hline Ximena & 31 años & Preparatoria & Comerciante & Soltera & Cree en Dios \\
\hline Susana & 46 años & Preparatoria & Asistente médico & Casada & Católica \\
\hline Gabriela & 47 años & Normal Superior & Subdirección en la SEP & Casada & Cree en Dios \\
\hline Beatriz & 43 años & Licenciatura & Periodista & Separada & Ninguna \\
\hline Elena & 49 años & Licenciatura & Ama de casa & Soltera & Católica \\
\hline Marcela & 46 años & Primaria & Ama de casa & Casada & Católica \\
\hline Victoria & 44 años & Normal Superior & Educadora & Casada & Ninguna \\
\hline Sara & 35 años & Primaria & Ama de casa & Casada & Católica \\
\hline Rosalba & 27 años & Licenciatura & Comerciante & Soltera & Ninguna \\
\hline Natalia & 51 años & Preparatoria & Ama de casa & Casada & Católica \\
\hline Marco & 33 años & Preparatoria & Jardinería & Casado & Cristiana \\
\hline Señor K & 30 años & Preparatoria & Danza & Unión Libre & Ninguna \\
\hline Eduardo & 39 años & Primaria & Chofer & Casado & Católica \\
\hline David & 52 años & Secundaria & Tapicero & Casado & Católica \\
\hline Javier & 52 años & Primaria & Carpintero & Casado & Católica \\
\hline Sergio & 51 años & Secundaria & Comerciante & Casado & Católica \\
\hline Omar & 63 años & Licenciatura & Sastre & Casado & Católica \\
\hline
\end{tabular}

SEP: Secretaría de Educación Pública

económicos (no alcanza el dinero, dificultades para cubrir las necesidades de la familia) y los legales (pago de impuestos, deudas en el banco, etcétera).

Para los médicos tradicionales, los problemas de tipo amoroso, familiar o económico tienen como consecuencia que las personas se depriman. Sin embargo, según la percepción de los curanderos, la gente se queja y los consulta para remediar sus conflictos amorosos y / o económicos y no por sentirse tristes, deprimidos y/o enojados. Es decir, la demanda de cura es para quitar el objeto o la causa del padecimiento, de lo que se deduce, con bastante claridad, que el demandante tiene la noción de que la tristeza, el enojo o la preocupación son sólo los síntomas de la enfermedad. Así lo señala el relato de Martha, quien se autocalifica como yerbera:

Una persona llegó conmigo, "señora yo vengo a que usted me ayude porque mi esposo me está engañando" y llorando me dice: "yo quiero deshacerme de él, yo quiero vengarme de él”. ¡Deténgase!, le digo, ¿a ver qué pasa? Me empezó a platicar toda su historia, empezó a llorar la mujer, empezó a desahogarse como una criatura, en- tonces le dije yo, mira qué te parece si hacemos una cosa, porque aquí conmigo no vas a encontrar nada de venganzas.

Yo estoy para ayudarlas, estoy para aconsejarlas si puedo, ya viniste, ya me explicaste, ya te desahogaste, ya me dijiste que es lo que te pasa, ¿qué te parece si hacemos una cosa? ¿Dígame señora que puedo hacer?” Mira yo te aseguro que si tu esposo te trató mal, no te dio dinero, sufren tus hijos. Si a ti te está haciendo todo eso, yo quiero que entiendas una cosa, tú no te lo vas a cobrar por tu propia mano, porque eso que a ti te está haciendo, otra persona lo va a cobrar, porque en este mundo todo lo pagamos, buenos y malos...

\section{El estrés, la tensión y los nervios}

El estrés, la tensión y los nervios también se encuentran entre los motivos primordiales por los cuales los curanderos perciben que son solicitados sus servicios y conocimientos. Señalan que este tipo de padeceres están asociados con una gran variedad de problemas emocionales y síntomas fisiológicos. En- 
tre los principales problemas emocionales asociados señalan las presiones y los miedos característicos del cotidiano vivir en las grandes ciudades. La desesperación, el consumo de drogas o alcohol por parte de algún miembro de la familia y las diferencias generacionales entre padres e hijos. Entre la diversidad de los componentes fisiológicos, con mayor frecuencia se mencionaron cansancio, dolor de cabeza, dolores estomacales, temblores en el cuerpo, presión arterial alta, inflamación del hígado, tensión muscular y que los nervios se encuentren fuera de su lugar, débiles o quebrantados. Cuatro pacientes mencionaron asistir con el curandero por problemas de este tipo.

La posibilidad de utilizar conceptos como nervios, tensión o estrés para diversos problemas está dada por la construcción cultural que existe, es decir, son conceptos que tienen un significado compartido dentro de la población citadina y que son del dominio popular. Lo anterior permite que exista una comunicación clara y directa entre el sanador y el usuario, de tal forma que si a una persona se le dice que está mal de los nervios, tensa o estresada, sabrá claramente cuál es su problema, y entenderá el tipo de tratamientos que debe recibir y las cosas que debe hacer para recuperar y conservar su salud. De la misma manera, permite a las personas situarse en un territorio entre lo normal y la locura. Es más, en la actualidad el estar nervioso, tenso o estresado podría hasta conceptualizarse dentro de lo normal. La sociedad se ha acostumbrado de tal forma a sufrir este tipo de padeceres que ya son parte de su vida cotidiana y no los califica como problemas de salud.

Padecimientos musculoesqueléticos y enfermedades relacionadas con los diversos sistemas que regulan el cuerpo

Dentro de la categoría de padecimientos muscoloesqueléticos, los médicos tradicionales incluyen las fracturas o quebraduras, las torceduras, los dolores musculares, los golpes, los calambres y las hernias, entre otros. Para cuatro de los pacientes, estos padecimientos fueron la razón para asistir con un sanador.

Dentro del grupo de enfermedades relacionadas con los diversos sistemas que regulan el cuerpo se incluyen enfermedades del riñón, males cardiacos, problemas respiratorios, problemas estomacales. También se consideran las enfermedades crónicas y el evitar las intervenciones quirúrgicas. Según los sanadores, la frecuencia con la que asisten personas con problemas muscoloesqeléticos o de los sistemas que regulan el cuerpo es inferior a la relacionada con problemas nerviosos, económicos, familiares o amorosos.

\section{Problemas espirituales*}

En lo que se ha denominado problemas espirituales, los médicos tradicionales agrupan las enfermedades relacionadas con el alma o espíritu. Para ellos, las enfermedades espirituales son el odio, el rencor, la ambición y los celos. También, la pérdida del alma queda dentro de esta categoría. Dicha pérdida puede presentarse por múltiples razones, entre las que destacan un accidente, un susto, una sorpresa, etcétera. Si la persona pierde el alma, se queda sin el control, ya que se le considera el elemento conductor del ser humano. Parten de la creencia, heredada de las doctrinas mesoamericanas y de las enseñanzas cristianas, de que cada persona tiene una alma singular y ésta es el principio vital de su ser. Así, los problemas espirituales sólo pueden ser curados por la misma persona que las padece y los curanderos sólo funcionan como "espejos" para ayudar al paciente a reconocer el origen de su problema anímico. Gustavo, médico tradicional, ofreció el siguiente relato:

Entonces el alma tiene otro tipo de enfermedades como son el odio, el rencor, la ambición, los celos, que esos no hay realmente quien los cure sino solamente uno. Podemos nosotros servir como espejos para reflejar los problemas de salud del alma, para que tú los reconozcas y para que tú los puedas solucionar, y hay métodos para dominarlos y hay métodos para alcanzarlos y comprenderlos y aceptarlos en el caso necesario y convertirlos en elementos que te permitan aprovechar estas fuerzas. En lugar de ser un elemento destructivo se convierte en un elemento positivo.

\section{Enfermedades "provocadas o puestas"}

De acuerdo con los informantes, las enfermedades provocadas o puestas también se encuentran entre los principales motivos de consulta. Se entienden como enfermedades provocadas a todas aquellas dolencias que son causadas por la envidia, la ira o los celos despertados en alguien. La persona que quiere hacer el daño o mal generalmente es muy cercana a la víctima (amigo, compañero de trabajo, vecino, familiar, rival, etc.). Para provocar el daño, se acude con un "brujo" o

\footnotetext{
* En todos los relatos de los médicos tradicionales los conceptos de espíritu o de alma se manejan como energía. El espíritu es el componente principal o la esencia de todo ser vivo, lo que lo hace único y cumple la función de animar o alimentar la vida.
} 
"curandero que usa magia negra", quien a través de encantos y conjuros envía el mal. La enfermedad así causada puede ser de cualquier género (desde un dolor de estómago hasta la locura) y tener síntomas muy diversos, pero generalmente son enfermedades sin una causa aparente.

Todos los médicos tradicionales entrevistados señalaron con insistencia que ellos no se dedican a provocar daño o enfermedades, que ellos sólo ayudan a quienes las padecen. Del mismo modo, ninguno de los pacientes asistió con un curandero para "hacer un trabajo" o "enviar un daño"; por el contrario: acudieron como víctimas del mal.

La mitad de los curanderos creen que la brujería como daño mágico no existe. En este sentido consideran que los individuos son convencidos por otras personas o por autosugestión de que les están haciendo un daño, y esta creencia es tan fuerte que ellos mismos se provocan la enfermedad. Esta es la opinión de Gustavo, médico tradicional:

Hay métodos de brujería, como dicen y le pongo dos comillas a los lados, que yo te trato de convencer, de hacerte un daño. Entonces te digo: "te voy a hacer un daño" y el día de mañana encuentras en tu casa una muñequita de trapo muy parecida a ti físicamente, atravesada con tres alfileres y tú sientes miedo. Ya le permitiste entrar a esto y va a entrar y va entrar y te va a destruir igual que la envidia que estamos comentando. Estos son métodos subliminales de enfermar a la gente. Tanto autosubliminarte como otro agente externo te puede subliminar".

Por su parte, la mayoría de los pacientes entrevistados consideran que sí es posible provocar enfermedades, desgracias o accidentes por medio de la brujería. Para ellos el daño no se realiza de manera fortuita, siempre existen motivos muy definidos, y las motivaciones principales son la envidia y los conflictos amorosos. Seis de los pacientes entrevistados (cuatro mujeres y dos hombres) fueron en algún momento víctimas de una brujería. Para los dos hombres y una de las mujeres, el mal fue causado a consecuencia de envidias, mientras que para las tres mujeres restantes la causa estuvo relacionada con conflictos amorosos. En esta sección sólo ejemplificaremos los casos relacionados con problemas de envidia, ya que en la siguiente categoría se verán con detenimiento los relacionados con conflictos amorosos. David recordó lo siguiente:

Mira yo tenía siete años. Yo vivía ahí en el Centro, en una vecindad fea, ahí se vivía lo peor, mariguana, co- caína, rateros, asesinos, de todo. Mi mamá tenía que trabajar todo el tiempo, nosotros, todos mis hermanos, nos quedamos ahí. Entonces, casi la mayoría de los que vivían ahí salieron malos, y a pesar de que nosotros no teníamos quien nos cuidara, no salimos malos. Pues así fue y nos agarraron envidia. Una vecina, no sabemos en que forma me hizo daño a mí, brujería. Los síntomas que yo sentía, empezaba yo a no sentirme de mi tamaño normal, hacerme como hormiguita. Y a mí ya me daba miedo de que me fueran a pisar. Yo no me hacía chiquito, en mi mente, mi mente era la que se hacía chiquita, me hacía chiquito yo. Me tapaban y no quería que me fueran a pisar, y por más que gritaba no me oían, entonces ya mi mente, ya estaba loca...

Padecimientos asociados con la identidad cultural de hombres $y$ de mujeres

Para los médicos tradicionales existen padecimientos que son más característicos de los hombres y otros más específicos de las mujeres. Esta división se realiza en un importante número de padecimientos; sin embargo, en este trabajo se incluyen solamente dos ejemplos que tienen que ver con la identidad cultural de ambos sexos: los conflictos amorosos en el caso de las mujeres y los problemas sexuales en el caso de los hombres.

\section{Conflictos amorosos}

Como ya se mencionó, los médicos tradicionales perciben los problemas o situaciones amorosas como una de las principales razones de consulta: conseguir pareja, conflictos matrimoniales o alejar a la amante, entre otras. Los sanadores consideran que este tipo de problemas afectan principalmente a las mujeres.

Tres de las usuarias entrevistadas informaron haber asistido con un curandero, vidente o brujo en busca de una solución para este tipo de problemáticas amorosas. Coincidieron en señalar que los problemas amorosos eran la consecuencia de una brujería o mal causado por otra mujer: la rival. Es decir, se tiende a culpar a un tercero. Sin embargo, el objeto al que va dirigido el daño no siempre es el mismo; en algunos casos el mal va dirigido a ellas, en otros, a sus hijos, hermanos o padres, y en un distinto tipo de situaciones hacia la pareja (novio, marido, etcétera).

Las que comentaron haber sido las receptoras directas del mal, sufrieron importantes desajustes de salud (malestares musculares, problemas estomacales, tumores, problemas neurológicos y parálisis facial). Algo similar sucedió con los familiares que fueron afectados por la brujería. 
Cuando la pareja fue la víctima del daño, la consecuencia fue distinta. No apareció enfermedad alguna. Sencillamente el novio se apartó, el marido se fue, la pareja cambió de actitud hacia ella o hacia los hijos o aportó menos dinero a la casa.

$\mathrm{Al}$ tratar las rupturas afectivas como consecuencia de una brujería, las mujeres son liberadas de cualquier culpa, ellas no tienen ninguna responsabilidad de lo sucedido. Del mismo modo, su hombre no las traicionó deliberadamente, fue víctima de una brujería o daño; por ello, en muchos casos de brujería, los hombres son perdonados y hasta protegidos. Victoria, una paciente, recuerda así su situación:

Yo sentía un dolor muy fuerte, aquí en la boca del estómago, un dolor terrible, a mí me despertaba el dolor, daba vueltas y vueltas, y me tomaba tés, tomaba pastillas. Fui a ver a una señora, una curandera que me limpió con un huevo y antes de romper el huevo lo oye y dice: "Este huevo tiene daño". Entonces, al romperlo, yo no podía creer lo que estaba saliendo en el huevo. Olía feo, empezó a desdoblarlo y empezó a ver que era una muñeca. Esa muñeca era yo y tenía alfileres en la parte del cuerpo donde me dolía, tenía yo tres alfileres. Cuando yo lo vi, me quedé muy sorprendida. La señora me dijo que este trabajo tiene más de tres años que lo hicieron, también limpiaron a mi esposo y ahí que tenía cuatro años que lo estaban trabajando, y ella nos dijo que era la misma persona que estaba trabajando a los dos, y era una mujer que andaba con mi esposo. Entonces como yo le estorbaba, entonces también me estaba trabajando a mí, trabajaba a mi hijo también, de esta forma nos trabajaba a toda la familia, de alguna manera quería la ruptura de la familia y que mi esposo pudiera irse a vivir con ella.

Problemas sexuales

De acuerdo con lo relatado por algunos los médicos tradicionales, un motivo importante de consulta por parte de los hombres son los problemas sexuales, sobre todo los relacionados con impotencia y eyaculación precoz. Sin embargo, ninguno de los pacientes manifestó buscar ayuda por un problema de tipo sexual.

A pesar de no contar con información suficiente, podríamos suponer que las preocupaciones sexuales están íntimamente ligadas con la identidad cultural. Por ejemplo, la impotencia sexual pone en riesgo la realización de la virilidad exigida por la cultura al hombre. Un hombre que pierde su potencia sexual, pierde gran parte de su hombría y con ello su poder sobre la mujer, así como el dominio ante otros hombres.

\section{Discusión}

A partir de los testimonios ofrecidos por los informantes, es posible señalar que se recurre a la medicina tradicional por problemas de diversas índoles (amorosos, conflictos familiares, apuros económicos, estrés, tensión, nervios, angustia espiritual, etc.) que cubren prácticamente toda la esfera vivencial del individuo y que abarcan espacios no fácilmente medicables. Las personas que acuden con los curanderos no solamente buscan curarse de sus males, también desean obtener consejos acerca de sus problemas personales. Así, la medicina tradicional es una opción más para hacer frente al sufrimiento humano.

Pero, ¿en qué radica la vigencia de la medicina tradicional?, ¿por qué la gente sigue recurriendo a la medicina tradicional, a pesar del crecimiento, avances tecnológicos y eficacia comprobable de la medicina científica? Las posibles razones son muchas; aquí intentaremos analizar sólo algunas:

1. Un elemento que resulta fundamental es que los representantes de la medicina tradicional no suponen la distinción entre los problemas del cuerpo y los de la mente. Para Cabieses, muchos galenos se han olvidado de que toda medicina se sustenta en el entramado psicosomático, es decir, en la unidad entre la mente y el cuerpo. ${ }^{28}$ Por el contrario, los médicos tradicionales saben muy bien la importancia de ambas partes, de hecho, es notable la maestría de los curanderos en su capacidad de relacionarse con sus pacientes y hurgar en el fondo de sus conflictos emocionales para luego canalizar sus motivaciones y sentimientos en aras de la curación. Aunado a lo anterior, Desjarlais y colaboradores consideran que el no hacer una división entre mente y cuerpo permite que las enfermedades, mal llamadas mentales, se perciban como parte de un complejo fenómeno de salud, evitando así que los enfermos sean estigmatizados, además de ampliarse la gama de posibles tratamientos y de incrementarse las esperanzas para la curación. ${ }^{29}$

2. Por otro lado, dentro de la medicina tradicional se concibe con claridad que el padecer es el resultado de la integración de la percepción que se tiene del cuerpo humano y sus molestias con la interpretación o significado que ha dicha percepción le confiere la historia personal del paciente, así como la cultura a la que pertenece. Al tener los médicos tradicionales esta clara concepción del padecer, entonces pueden entender y respetar con mayor facilidad la manera en que cada paciente 
nombra y vive su problema de salud, independientemente de que sea o no enfermedad.

3. Del mismo modo, tratar algunos padecimientos como consecuencia de brujería o daño facilita que los pacientes se liberen de cierta carga de culpabilidad, ya que el responsable de su malestar es un tercero (quien provocó el daño). Aunado a lo anterior, el paciente recibe una explicación sobre su problemática que le es coherente, clara y acorde con sus creencias, lo que resulta de gran utilidad en problemas de salud difíciles de diagnosticar, en padecimientos crónicos donde la cura es casi imposible o en situaciones donde resulta necesario una modificación en la vida social del paciente.

4. Otra de las circunstancias que explican la continuidad de la medicina tradicional en espacios urbanos como el estudiado en este articulo está relacionada con la capacidad de los curanderos para adaptarse a las necesidades cambiantes de la sociedad. Han aprendido que dada la diversidad de problemas de salud que sufre la población, es necesario cubrir al mismo tiempo diferentes roles (consejero de amor y de leyes, nutriólogo, brujo, guía espiritual, etc.). Autores como Press, ${ }^{15}$ Campos ${ }^{12}$ y Prophet $^{30}$ consideran que la medicina tradicional urbana busca, a través de esta nueva combinación de roles, mantener una forma de identidad frente a los múltiples procesos de cambio.

5. Finalmente, una de las diferencias fundamentales a favor del empleo de la medicina tradicional consiste en ocuparse y tratar-sobre todo con medios simbólicos- lo que Kaja Finkler ha llamado las lesiones de la vida. ${ }^{31}$ Las define de la siguiente manera: "Por lesiones de la vida entiendo las adversidades percibidas de la existencia, incluyendo relaciones sociales hostiles y contradicciones no resueltas, en las que la persona está profundamente involucrada, y que atormentan a su ser y causan resentimiento a lo largo del tiempo, lo que produce una multitud de síntomas o padecimientos que no atentan contra la vida. Las lesiones de la vida implican la expresión corporal de la adversidad, de las contradicciones del existir y recrean en el mundo interno del cuerpo las paradojas y desorden del mundo externo. Siguiendo a Merleau-Ponty, ${ }^{30}$ quien propuso que el cuerpo se comunica con el mundo y que el mundo se expresa a través del cuerpo, Kleinman señala: ${ }^{17}$ "El cuerpo siente y expresa los problemas sociales. El cuerpo es un mediador de la estructura y el significado social, y hace a éstos parte de la fisiología. Las lesiones de la vida pene- tran en el cuerpo, de manera semejante a los agentes patógenos o a las lesiones anatómicas, pero no son mortales, como tienden a serlo los infartos del corazón o el cáncer. Las lesiones de la vida de una persona reflejan su historia o biografía, inscrita en el cuerpo y manifestada por angustia, dolor generalizado en la totalidad del mismo, y por síntomas cuya etiología no se puede dilucidar, pero que no amenazan la sobrevivencia. Esta sintomatología, con frecuencia, no se puede traducir en los signos en los que se basan los diagnósticos médicos y no se manifiestan en los equipos e instrumentos diagnósticos de la medicina. Las inscripciones talladas en el cuerpo por las lesiones de la vida hacen que las personas estén dolorosamente conscientes de su cuerpo y de estar enfermas".

Los conjuntos de signos y síntomas somáticos que son las manifestaciones de las lesiones de la vida rara vez encajan dentro de los cuadros clínicos que los médicos científicos están capacitados para comprender, diagnosticar y tratar. Molestias aisladas o en conjunto, del tipo de cansancio, mal sabor de boca, dolor de espalda y otras, que no se integran de manera coherente, desde el punto de vista de la clínica de la biomedicina, y tampoco hallan su contraparte en los resultados de los exámenes de laboratorio y gabinete, suelen ser atribuidas a la imaginación de los pacientes. En cambio, los médicos tradicionales actúan ante ellos, muchas veces ponen atención, discuten y ofrecen soluciones a las quejas de sus pacientes respecto a sus problemas de la vida cotidiana, entre los que sobresalen las relaciones dentro de y con la familia, los vecinos o los compañeros de trabajo, fuentes de conflictos habitualmente mal resueltos y capaces de ser percibidos como amenaza y causa de angustia durante largo tiempo. Con frecuencia la medicina tradicional ofrece su apoyo simbólico en forma de amuletos, oraciones, rutinas de vida o ceremonias - por ejemplo, las limpias- donde el paciente siente bienestar y, cuando menos, considera que está realizando acciones concretas para resolver su problema.

De lo anterior podemos concluir que hay múltples razones válidas para explicar no solamente la supervivencia de la medicina tradicional, sino además su éxito en el mundo actual, donde la ciencia y la tecnología han obtenido resultados sobresalientes, pero, a cambio, la biomedicina ha olvidado la complejidad de la vida humana cotidiana y el valor de la atención personal, la compasión, la comprensión, la empatía y el uso del mundo simbólico para la solución de los conflictos y problemas de salud propios de la humanidad. 


\section{Agradecimientos}

A las personas entrevistadas que compartieron sus saberes y experiencias para hacer posible este trabajo.

\section{Referencias}

I. Organización Panamericana de la Salud. Sistemas de salud tradicionales en América Latina y el Caribe: Información Base. Informe técnico. Washington: Organización Panamericana de la Salud, 1999. 2. Organización Mundial de la Salud. Estrategia de la OMS sobre medicina tradicional 2002-2005. Ginebra: Organización Mundial de la Salud, 2002.

3.Viesca C. Etnomedicina e investigación interdisciplinaria. En:Vargas L; Viesca C, ed. Estudios de Antropología Médica. México: Universidad Nacional Autónoma de México, 1986:13-20.

4. Alba D, Pinzón M. Reflexiones metodológicas para abordar la dimensión cultural en el desarrollo local.Actas del VIII Congreso de Antropología en Colombia, Universidad de Colombia, 1997 dic 5-7; Sta Fe, Bogotá. Colombia.

5. Eisenber G, Kessler D, Foster C, Norlock F, Calkins D, Delbanco T. Unconventional medicine in the United States: prevalence, costs, and patterns of use. N Engl J Med 1993;328:246-252.

6. Lazar J, O'Connor B. Talking with patients about their use of Alternative Therapies. Prim Care 1997;24: 699-714.

7. Eisenberg D, Davis R, Ettner S, Appel S, Wilkey S,Van Rampay M et al. Trends in alternative medicine use in the United States, 1990-1997. Results of a follow-up national survey. JAMA 1998;280:1569-1575. 8. Astin, J.Why patients use alternative medicine: Results of a national study.JAMA 1998; 279:1548-1553.

9. Keesler R, Soukup J, Davis R, Foster D, Wilkey S. The use of complementary and alternative therapies to treat anxiety and depression in the United States.Am J Psychiatry 200 I; I58:289-294. 10. Zolla C, del Bosque S, Mellado V, Tascón A, Maque C. Medicina tradicional y enfermedad. En: Campos R, ed. La antropología médica en México,Tomo 2. Antologías Universitarias, México: Instituto Mora, 1992:71-104.

II. Lozano S. Curanderismo: demystifying the health beliefs and practices of elderly Mexican-Americans. Health Soc Work 1995;20:247-253.

12. Campos R. Nosotros los curanderos. México: Editorial Nueva Imagen, 1997.

13. Berenzon S. La medicina tradicional urbana como recurso alternativo para el tratamiento de problemas de salud mental (tesis). México: Universidad Nacional Autónoma de México, 2003.

14. Redfield R. Yucatán. Una cultura de transición. México: Fondo de Cultura Económica, 1943
15. Press I. Urban folk medicine: a functional overview. Am Anthropo 1978;80:7|-84

16. Trotter R, Chavira J. Curanderismo: Mexican American folk health system. Georgia: University of Georgia Press, 1981.

17. Kleinman A. Social origins of distress and disease: depression, neurasthenia and pain in Modern China. New Haven:Yale University Press, 1986.

18. Mercado F: Entre el infierno y la gloria. La experiencia de la enfermedad crónica en un barrio de Guadalajara. Guadalajara: Universidad de Guadalajara, 1996.

19. Sierra F. Función y sentido de la entrevista cualitativa. En: Galindo J. coord. Técnicas de investigación en sociedad, cultura y comunicación. México:Addison Wesley Longman, 1999:277-34I.

20. Glaser B, Strauss A. The discovery of grounded theory. Chicago: Aldine, 1967.

21.Trotter R, Medina-Mora M. Qualitative methods. En:World Health Organization, ed. Guide to drug abuse epidemiology. Washington:WHO, 2000:124-136

22. París M. Investitgación cualitativa en salud: superando los horrores metodológicos. En: Bronfman M, Castro R, coord. Salud, cambio social y política. Perspectivas desde América Latina. México: EDAMEX, 1999:35-47. 23. Taylor S, Bodgan R. Introducción a los métodos cualitativos de investigación. Buenos Aires: Paidós Studio, 1986

24. Galindo J. Encuentro de subjetividades, objetividad descubierta. La entrevista como centro del trabajo etnográfico. Estudios sobre las culturas contemporáneas 1987;3:151-183.

25. Kvale S. InterWiews: An Introduction to qualitative research interwiewing. Londres: Sage Publications, 1996.

26. Castro R, Bronfman M. Problemas no resueltos en la integración entre métodos cualitativos y cuantitativos en la investigación social en salud. En: Bronfman M, Castro R, coord. Salud, cambio social y política. Perspectivas desde América Latina. México: Instituto Nacional de Salud Pública, 1999:49-64.

27. Alonso L. Sujeto y discurso: "El lugar de la entrevista abierta en las prácticas de la sociología cualitativa”. En: Delgado J y Gutiérrez J, eds. Métodos y técnicas de investigación en ciencias sociales. Madrid: Síntesis, 1995: 225-240.

28. Cabieses F. La articulación de las medicinas tradicionales con la medicina oficial. Anales de la Facultad de Medicina de la Universidad de San Marcos 1996;57:1-7.

29. Desjarlais R, Eisenberg L, Good B, Kleinman A. Salud mental en el mundo: problemas y prioridades en poblaciones de bajos ingresos. Washington: Oxford University Press, 1997.

30. Prophet S. Alternative Medicine: growing trend for the new millenium. J AHIMA 1998;66-76.

3I. Finkler K. Physicians at work, patients in pain. Biomedical practice and patient response in Mexico. North Carolina: Carolina Academic Press, 200I. 